\title{
Erratum
}

Notfall Rettungsmed 2021 $\cdot 24: 98$

https://doi.org/10.1007/s10049-020-00809-1

Online publiziert: 30 . November 2020

(c) Springer Medizin Verlag $\mathrm{GmbH}$, ein Teil von Springer Nature 2020

\section{Adler ${ }^{1,2} \cdot$ R. Stangl ${ }^{2}$}

${ }^{1}$ Klinik III für Innere Medizin - Allgemeine und interventionelle Kardiologie, Elektrophysiologie, Angiologie, Pneumologie und internistische Intensivmedizin, Herzzentrum der Universität zu Köln, Köln Deutschland

${ }^{2}$ Institut für Schutz und Rettung, Berufsfeuerwehr Köln, Köln, Deutschland

\section{Erratum zu:}

Kreislaufstabilisierung im Notfall. Möglichkeiten der medikamentösen Kreislaufstabilisierung im Rettungsdienst und in der Notaufnahme

\section{Erratum zu:}

Notfall Rettungsmed 2020

https://doi.org/10.1007/s10049-020-

00778-5

Im ursprünglich veröffentlichten Artikel ist in der Spalte Kardiopulmonale Reanimation der Tab. 1 ein Fehler aufgetreten. Bitte beachten Sie die nun korrigierte Fassung.

\section{Korrespondenzadresse}

\section{Dr. med. C. Adle}

Klinik III für Innere Medizin - Allgemeine und interventionelle Kardiologie, Elektrophysiologie, Angiologie, Pneumologie und internistische Intensivmedizin, Herzzentrum der Universität zu Köln

Kerpener Str. 62, 50937 Köln, Deutschland christoph.adler@uk-koeln.de 